\title{
ScaleBrewer: A Visual Design Tool for Graphical Customization of Map Scale
}

\author{
Meilin Liu ${ }^{\mathrm{a}, \mathrm{b}}$, Xi Tang ${ }^{\mathrm{a}, \mathrm{b}, \mathrm{c} *}$ \\ ${ }^{a}$ Key Laboratory of Geographic Information Sciences (Ministry of Education), East China Normal University, Shanghai, 200241, China; \\ ${ }^{b}$ School of Geographic Sciences, East China Normal University, Shanghai, 200241, China \\ ${ }^{c}$ Institute of Cartography, East China Normal University, Shanghai, China \\ * Corresponding author, e-mail: xtang@geo.ecnu.edu.cn
}

Keywords: Map scale, Visual design, Map deformation, Map decoration

\begin{abstract}
:
Introduction: The well-developed version of various mapping software such as ArcGIS and QGIS has greatly reduced the time and other cost of map production, and becomes more accessible to non-specialized users. The result leads to a growing diversity of map styles. However, most mapping software focuses on how to speed up mapping tasks. In terms of the visual optimization of maps, they mainly revolve around color scheme and symbol design with less attention to assistant graphic elements such as scale, north arrow, graticule, etc. As especial one of map decoration elements, the graphical design of map scale not only affects the users' spatial sense, but also plays a vital role in map visual balance adjustment. So it would be an important issue worth studying that how to further enrich and customize the style of map scale form, and how to express map scale more scientifically.
\end{abstract}

By summarizing and categorizing map scale forms, we deconstruct the scale components to bars, nodes, and annotation fonts and their unit in order to create new visual styles for them. Meanwhile, we put a necessary premium on the map deformation caused by different projections or geometric perspective ways, which gives neglected potential impacts on map scale configuration. Then we produce a visual design tool for graphical customization of map scales, especially under several common projection types. This tool can help users to achieve process-oriented and personalized design paradigm and to innovate map scale style, while it can make response to the form matching requirements from deformed maps. All design results above are showing in a "what you see is what you get" way. It will effectively enrich the library of map scale options, and could be an alternative teaching prop to illustrate the correct cognitive relationship between map space and real space.

Form analysis and visual design of map scales: Although most of map scales occupy small area on the map, they contain a variety of primitives such as points, lines, surfaces, and fonts, which need to be designed with a reasonable placement and layout in mind. We decompose the scale graphics into four parts: bars, nodes, numbers and units. Then we select seven variables of shape, color, texture, frequency, size, position, and interval to give a collection of their illustrative plates with varied graphical solutions.

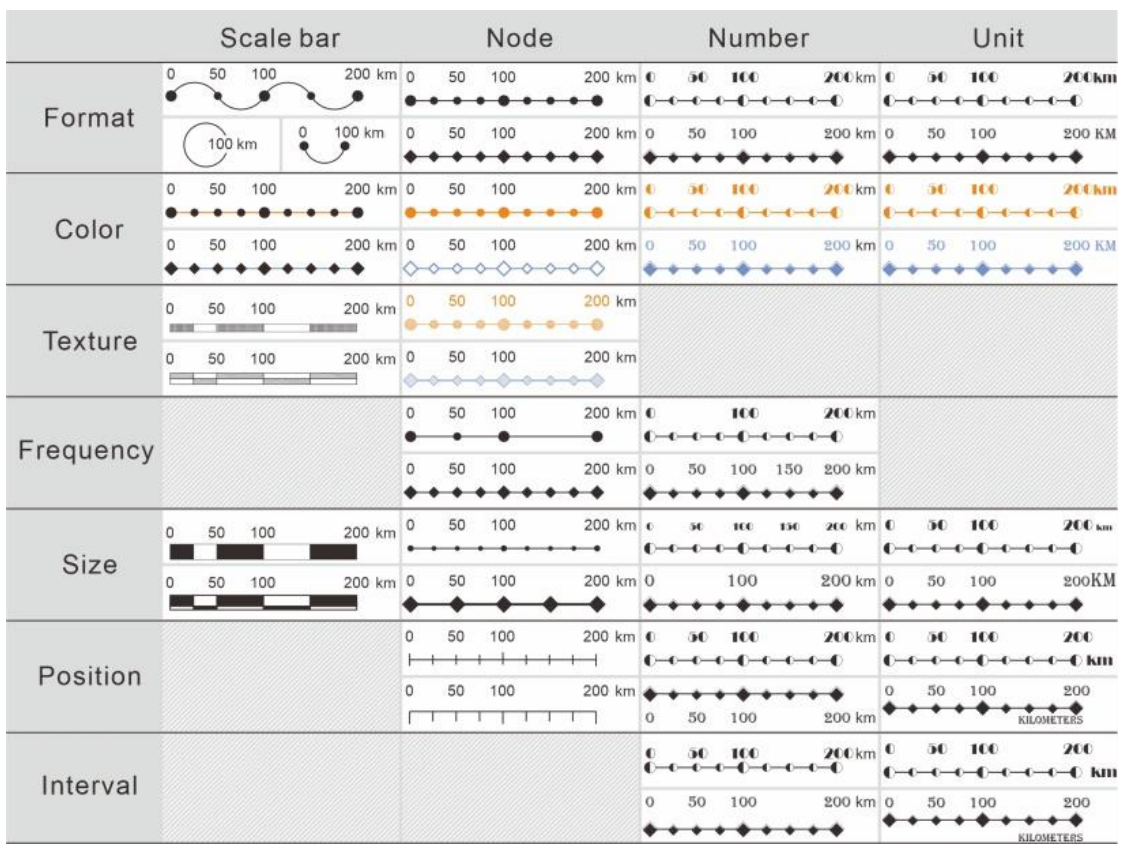

Figure 1. Design matrix of map scales based on visual variables and primitives. 
Scale representation with graphic variation under map deformation: As the mathematical basis of maps, the local scale is influenced by the map deformation. In this paper, map deformation is divided into two main categories: one is caused by map projections, the other is caused by special presentation of maps such as geometric perspectives.

Features on the surface of a sphere or an ellipsoid undergo distortions when they are projected onto a plane. It is necessary to have a precise definition of the amount of distortion that has resulted. Taking several common map projections such as equidistant cylindrical projection, azimuthal equidistant projection and equidistant conic projection as examples, we use Scale factor (SF) to represent the scale change along meridians and parallels. In addition to the linear-segment scale, we also use the arc-segment form according to the graticules shape of normal azimuthal or conic projection conditions.

When a large-scale map is presented in some geometric perspective condition, the scale change may be simultaneous along both sides of the rectangular grids. it is more difficult to judge relative distances than to do it on a projected map with deformation occurs only on one principal direction. The 'Grid Scale' can be designed to solve this problem.

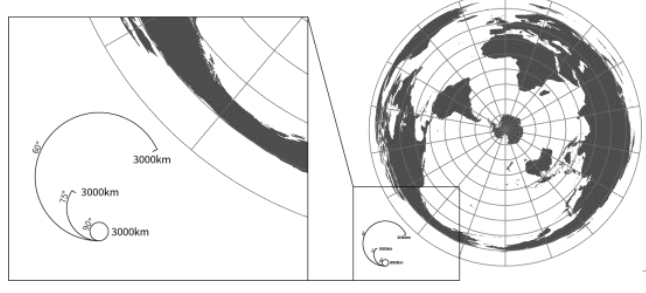

Figure 2. Arc-segment scale.

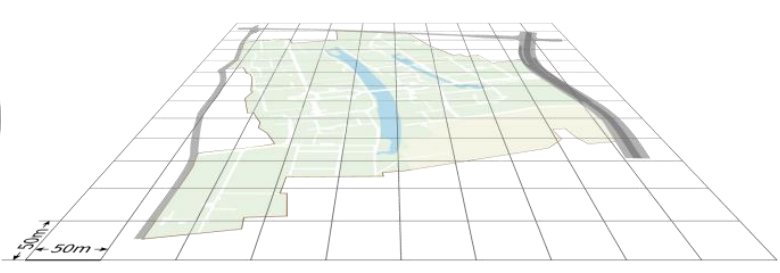

Figure 3. Grid scale.

ScaleBrewer: a prototyping tool for map scale design: Finally, we develop a visual design prototyping tool for map scale customization based on HTML and CSS. Its main interface consists of four modules: scale settings, scale preview, map display and projection introduction. Users can click on buttons of each module to select the responsive element attributes and customize the scale style they need. The current scale style can be previewed on the interface bottom (left one in blue colour). Besides, the standard map scale under the current projection is displayed by default (right one in blue colour). However, if user inputs some coordinate latitude and longitude, the default scale changes to the actual scale of the selected location. When the 'start' button at the bottom is clicked, an active scale segment appears (in red colour) and will move following the mouse position. This scale segment's distance value is fixed but its visual length changes in real time. On the right information panel, there is a brief description of the current projection with a hyperlink to its Wikipedia Term.

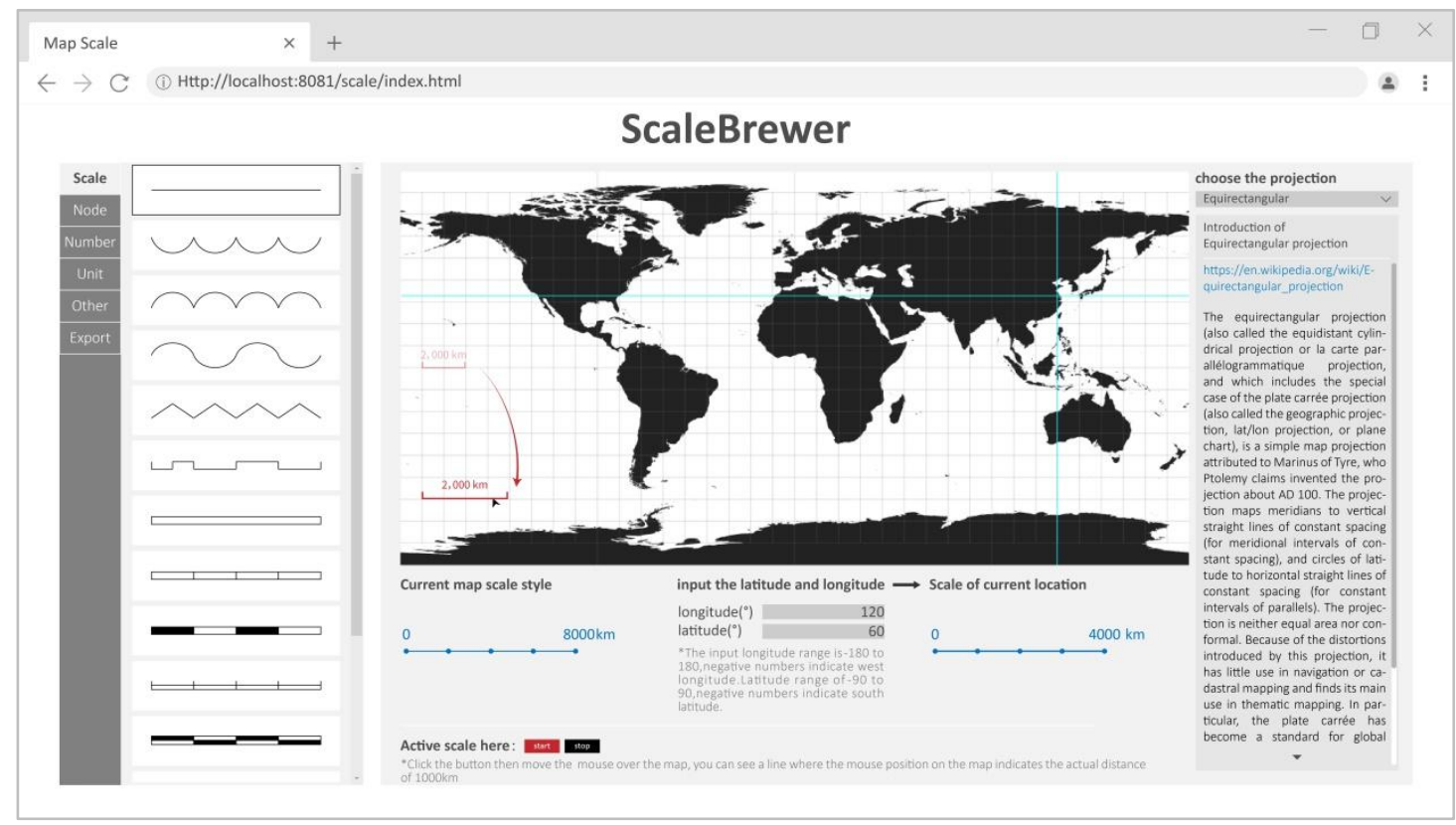

Figure 4. Main Interface of 'ScaleBrewer'

\section{Acknowledgements:}

The work was supported by the National Natural Science Foundation of China (grant number 41871374); 